\title{
Der strafrechtliche Schutz bei parteiinternen Wahlen
}

\author{
Marcus Hausen
}

Im Zuge der Diskussion über die strafrechtlichen Konsequenzen der Abgabe eines fehlerhaften Rechenschaftsberichts einer politischen Partei in den vergangenen Jahren ${ }^{1}$ ist die Frage anderer Strafbarkeit im Zusammenhang mit Parteiaktivitäten in den Hintergrund geraten. So gibt es bislang keine eingehende juristische Auseinandersetzung damit, ob und gegebenenfalls welches Fehlverhalten bei Wahlen innerhalb einer Partei zu strafrechtlichen Konsequenzen führen kann. ${ }^{2}$ Dabei tauchen Schlagzeilen über manipulierte Wahlen bei Parteien häufiger auf ${ }^{3}$, und der Bundestag sowie die Landesparlamente haben nach der Entscheidung des Bundesverfassungsgerichts vom 20. Oktober $1993^{4}$ die Anforderungen an die Aufstellungen von Bewerbern zu öffentlichen Wahlen erweitert.

Diese Anforderungen bergen Schwierigkeiten, mit denen sich selbst erfahrene Juristen schwer tun. Deutlich wird dies bei Abgabe der Versicherung an Eides statt, die über den Ablauf einer Aufstellungsversammlung nach $\$ 21$ Abs. 6 Satz 2 BWahlG in Verbindung mit

1 Vgl. Jörn Ipsen, Einführung, in: ders. (Hrsg.), 40 Jahren Parteiengesetz, Osnabrück 2009, S. 9 12, S. 10. Zu Fragen der Parteienfinanzierung und des Strafrechts siehe insbesondere Frank Saliger, Parteiengesetz und Strafrecht - Zur Strafbarkeit von Verstößen gegen das Parteiengesetz insbesondere wegen Untreue gemäß $\$ 266$ StGB, Tübingen 2005; darüber hinaus Jutta Faust, Zur möglichen Untreuestrafbarkeit im Zusammenhang mit Parteispenden, Frankfurt am Main 2006; Arne Krumbolz, Finanzierung und Rechnungslegung der politischen Parteien und deren Umfeld, Baden-Baden 2010; Martin Morlok, Parteienfinanzierung im demokratischen Rechtsstaat, Berlin 2009; Kerstin Muthers, Rechtsgrundlagen und Verfahren zur Festsetzung staatlicher Mittel zur Parteienfinanzierung, Osnabrück 2004; Andreas Schalück, Parteienfinanzierung: Die Reform des Parteienfinanzierungsgesetzes - „Pflege der politischen Landschaft“ oder illegale Einflussnahme, Bad Honnef 2006; Christian Sirch, Die Strafbarkeit der Parteispendenakquisition, Frankfurt am Main 2008.

2 Dominik Härtel (Wahlstraftaten, Frankfurt am Main 2006, S. 195 f.) widmet dem Thema Wahlstraftaten und Parteien knapp zwei Seiten unter dem Gesichtspunkt, ob Wahlstraftaten um parteiinterne Abstimmungen erweitert werden sollten. Die übrige Literatur zu diesem Thema stammt aus den 1950er und 1960er Jahren. Vgl. Kurt Trinkaus, Die Wahldelikte im geltenden deutschen Strafrecht, Bonn 1955; Gerhard Wolf, Straftaten bei Wahlen und Abstimmungen, Bonn 1961; Ernst Heinrich Kirschner, Die Wahldelikte im geltenden Recht und im Entwurf 1962, Köln 1964; Niederschriften über die Sitzungen der Großen Strafrechtskommission, Bonn 1960, Bd. 13, S. $289 \mathrm{ff}$.

3 Siehe beispielsweise Björn Hengst, Seltsamer Masseneintritt irritiert Hamburger CDU, in: Spiegel Online vom 23. Dezember 2005, http://spiegel.de/politik/deutschland/0,1518,392112,00.html; „CDU - Verschwörung auf Kölsch“, in: Spiegel vom 13. Februar 2006, S. 21; Arne Boecker, Chaos-Wahl bei Hamburgs SPD - Der Staatsanwalt ermittelt, in: sueddeutsche.de vom 26. Februar 2007, http://www.sueddeutsche.de/politik/chaoswahl-bei-hamburgs-spd-der-staatsanwaltermittelt-1.807934; Lisa Erdmann, Niels Annen verliert sein Direktmandant, in: Spiegel Online vom 15. November 2008, http://www.spiegel.de/politik/deutschland/0,1518,590683,00.html; Bernd Dörries, Die Junge Skandal-Union, in: SZ vom 21. Oktober 2010, S. 6; Kristian Frigelj, Vorsicht, Wahl-Nomaden, in: Die Welt vom 13. Februar 2011, S. NRW4; Sandra Kaiser / Marita Jüngst / Clemens Henle / Mike Mic, Wahlnomaden - Kein Einzelfall, in: Rheinische Post online vom 3. Februar 2011, http://www.rp-online.de/niederrhein-nord/duisburg/nachrichten/wahlnomaden-kein-einzelfall-1.1191787 (Abruf jeweils am 1. Oktober 2011). 
$\$ 156$ StGB abzugeben ist. Der Inhalt der eidesstaatlichen Versicherung erstreckt sich auf nahezu alle Einzelheiten der Aufstellungsversammlung ${ }^{5}$, selbst mögliche kleine Fehler bei den Verfahrensabläufen können zu einer falschen Versicherung an Eides statt führen. So ist es für eine Verletzung des Grundsatzes der geheimen Wahl zum Beispiel ausreichend, wenn die nicht zwingend zu nutzenden Wahlkabinen wegen der Überfüllung des Raumes nur schwer oder sogar gar nicht zu erreichen sind. Gerade bei neuen Parteien führt mangelnde organisatorische Erfahrung schnell zu Auseinandersetzungen und darauf basierendem Fehlverhalten.

Die etablierten Parteien gehen außerdem vermehrt dazu über, das Delegiertensystem auf kommunaler Ebene, zum Teil motiviert durch sinkende Mitgliederzahlen, durch Mitgliederversammlungen zu ersetzen. Dabei kommt es schnell zu Wahlen und Aufstellungsversammlungen mit über 900 Stimmberechtigten. Bei dieser Größenordnung mit dem dazugehörenden Zeitdruck bei der Einlasskontrolle und den Auszählungen können leicht Fehler passieren. Zum Teil werden diese aber auch bewusst gemacht, um gezielt Personen zu einem Abstimmungserfolg zu verhelfen.

\section{Fallkonstellationen}

Ziel einer vorsätzlichen Manipulation einer parteiinternen Wahl ist es in aller Regel, dem bevorzugten Kandidaten eine Abstimmungsmehrheit zu sichern. Dazu gibt es unterschiedliche Möglichkeiten, die auch davon abhängen, ob die Wahl in einem Mitglieder- oder einem Delegiertensystem durchgeführt wird.

Bei letzterem stimmen von einer unteren Parteiebene gewählte Delegierte über die jeweils zu wählenden Mandats- beziehungsweise Funktionsträger ab. Bei Mitgliederwahlen handelt es sich in aller Regel um Versammlungen, auf denen grundsätzlich alle Mitglieder der örtlichen Parteigliederung abstimmungsberechtigt sind. ${ }^{6}$ Zumeist erscheinen jedoch nicht alle Delegierten und bei weitem auch nicht alle Mitglieder bei den Versammlungen. Dennoch findet häufig - auch aufgrund eines kurzfristigen Andrangs - bei der Ausgabe der Wahlunterlagen nur eine unzureichende Personenkontrolle statt, so dass es vorkommen kann, dass Wahlunterlagen an Nicht-Stimmberechtigte ausgegeben werden oder jemand die Unterlagen doppelt erhält. Durch eine nicht sorgfältige Personenkontrolle wird somit die Möglichkeit eröffnet, das Ergebnis einer Wahl zu „verfälschen“.7

Bei Delegiertenwahlen kann ein Bewerber zudem dadurch unterstützt werden, dass die Aufnahmeformulare gefälscht oder Personen vorgeschoben werden, die Mitglied werden,

5 Vgl. Wolfgang Schreiber, Bundeswahlgesetz, Köln u.a. 2009, \$ 21 Rdnr. 43.

6 Im Unterschied zu den rein parteiinternen Wahlen (Vorstands- und Delegiertenwahlen) sind bei der Aufstellung von Bewerbern für öffentliche Wahlen nur die Parteimitglieder stimmberechtigt, die ihren Wohnsitz in dem jeweiligen Wahlbezirk haben, $\$ 21$ Abs. 1 Satz 2 BWahlG. Dieser ist nicht immer in den örtlichen Grenzen identisch mit denen der örtlichen Parteigliederung. Siehe dazu BVerfGE 89, S. 243 ff., LS 3a, 256 f.; Il Sun Hong, Verfassungsrechtliche Probleme der innerparteilichen Kandidatenaufstellung für die Wahl zum Deutschen Bundestag, Baden-Baden 2005, S. 26; Wolfgang Schreiber, a.a.O. (Fn. 5), \$21 Rdnr. 11, 14.

7 Selbst im Deutschen Bundestag sind Abstimmungsfehler nicht zu vermeiden, beispielsweise bei der Abstimmung über die Mehrwertsteuererhöhung am 26. Mai 2006. Vgl. Jörg Kürschner, Streit beigelegt - Nach der Abstimmungspanne, in: Das Parlament vom 6. Juni 2006, S. 2. 
an der Abstimmung selber aber verlässliche Dritte teilnehmen. Darüber hinaus erhält die aufnehmende Untergliederung auf diese Weise mehr Delegierte zur Unterstützung des jeweiligen Kandidaten. ${ }^{8}$

Natürlich ist auch der gegenteilige Fall denkbar, dass der zuständige Vorstand sich weigert, über Mitgliedsanträge zur Neuaufnahme zum jeweiligen Stichtag zu entscheiden, so dass es auch nicht zu einer Erhöhung der Delegiertenzahlen kommt.

Eine weitere Möglichkeit der „Verfälschung“ des Wahlergebnisses besteht darin, ganze Urnen sowie einzelne Stimmzettel zu verändern (beispielsweise diese ungültig zu machen oder weitere Kandidaten bei Blockwahlen anzukreuzen), auszutauschen oder neue hinzuzufügen.

\section{Rechtliche Differenzierung parteiinterner Wablen}

Parteiinterne Wahlen lassen sich rechtlich in Vorstands- und Delegiertenwahlen sowie Kandidatenaufstellungen differenzieren. Während die Rechtsgrundlage für Vorstands- und Delegiertenwahlen in $\$ \$ 9$ ff. PartG in Verbindung mit den allgemeinen Regelungen des Vereinsrechts nach $\$ \$ 21 \mathrm{ff}$. BGB zu finden ist, ist das Aufstellungsverfahren von Bewerbern zu öffentlichen Wahlen gemäß $\$ 17$ PartG auch in den korrespondierenden Vorschriften des Wahlrechts geregelt. Diese Regelungen, insbesondere $\$ 21$ BWahlG, stellen deutlich höhere formelle Anforderungen an den Ablauf einer solchen parteiinternen Versammlung. ${ }^{9}$

Die Besonderheit der Kandidatenaufstellung im Vergleich zu sonstigen parteiinternen Wahlen und Abstimmungen wird vornehmlich dadurch deutlich, dass bei der Benennung von Bewerbern für Wahlen von vornherein ein strafrechtlicher Schutz besteht durch die gemäß $₫ 21$ Abs. 6 Satz 2 BWahlG abzugebende strafbewehrte eidesstattliche Versicherung. Die weiteren strafrechtlichen Konsequenzen sollen im Folgenden geklärt werden.

\section{Vorstands-und Delegiertenwahlen}

Der strafrechtliche Schutz bei parteiinternen Vorstands- und Delegiertenwahlen kann sich nur aus den allgemeinen Strafrechtstatbeständen ergeben, da sich in den Wahlgesetzen selber keine Normen finden, die auf derartige Fälle anwendbar sein könnten. ${ }^{10}$ Die $\$ \$ 107 \mathrm{ff}$. StGB sind gemäß $\$ 108 \mathrm{~d}$ StGB nicht auf Vorstands- und Delegiertenwahlen anwendbar.

8 Siehe $\$ 13$ Satz 2 des Gesetzes über die politischen Parteien (PartG), wonach sich die auf einen Gebietsverband entfallenden Vertreter in erster Linie nach der Zahl der vertretenen Mitglieder bemessen.

9 Die Vorschriften des Bundes und der Länder zu Wahlen gleichen sich im Grundsatz. Daher wird im Folgenden auf die Vorschriften des Bundeswahlgesetzes und der Bundeswahlordnung abgestellt.

10 Die einzige nebengesetzliche Strafrechtsnorm ist $\$$ 49a BWahlG. Dieser sieht in Abs. 1 lediglich vor, dass ordnungswidrig handelt, wer ohne wichtigen Grund das Ehrenamt als Beisitzer in den Wahlausschüssen oder als Mitglied eines Wahlvorstands ablehnt oder sich ohne genügende Entschuldigung den Pflichten eines solchen entzieht oder Ergebnisse von Wählerbefragungen nach der Stimmabgabe über den Inhalt der Wahlentscheidung vor Ablauf der Wahlentscheidung veröffentlicht. 


\subsection{Fälschung von Aufnahmeanträgen}

Für die Fälle der Fälschung von Aufnahmeanträgen sind fraglos die Urkundenfälschungsdelikte des $\$ 267$ StGB („Herstellen und Gebrauchmachen einer unechten Urkunde“) einschlägig. Indem ein Aufnahmeantrag für eine Partei einer Person zugeschrieben wird, den diese gar nicht abgegeben hat und von dem sie gegebenenfalls auch gar keine Kenntnis hat, wird eine unechte Urkunde hergestellt, von der durch das Vorlegen bei der zuständigen örtlichen Parteigliederung Gebrauch gemacht wird. Fraglich ist jedoch, ob und wieweit der Aufnahmeantrag einer dritten Person zuzurechnen ist. Hierfür kommt es auf die Umstände des Einzelfalls an.

\subsection{Nicht erfolgte Aufnahme in eine Partei}

Der spiegelbildliche Fall, das Hinauszögern einer Aufnahme durch den zuständigen Vorstand, ist nicht strafbewehrt. Hieran kann auch kein rechtspolitisches Interesse bestehen, da nicht jede Tagesordnung einer Sitzung des zuständigen Parteigremiums unter dem Damokles-Schwert strafrechtlicher Ermittlungen wegen möglicher nicht aufgenommener Mitglieder stehen kann. Zudem besteht über die parteiinternen Schiedsgerichte oder gegebenenfalls auch die ordentlichen Gerichte grundsätzlich die Möglichkeit, Rechtsschutz zu erlangen, wenn ersichtlich ist, dass Aufnahmeanträge bewusst über längere Zeit zurückgehalten werden.

\subsection{Erschleichen von Wahlunterlagen}

Für das „Erschleichen“ der parteiinternen Abstimmungsunterlagen könnten ebenfalls die Urkundendelikte nach $\$ \$ 267 \mathrm{ff}$. StGB in Betracht kommen. Ob und wie, kann allerdings nur anhand des Einzelfalls beurteilt werden, da es entscheidend davon abhängt, wie die Ausgabe der Wahlunterlagen organisiert wird, das heißt, ob allein die Angabe eines Namens ausreicht, oder ob die Einladung oder der Personalausweis vorgezeigt werden muss, um die Wahlunterlagen zu erhalten. Weiterhin ist zu berücksichtigen, wie seitens der Handelnden vorgegangen wird, also ob falsche Dokumente, beispielsweise eine im Anschriftenfeld veränderte Tagungseinladung, verwandt werden.

Vermögensdelikte, beispielsweise $₫ 265$ a StGB, sind nicht einschlägig, selbst wenn ein Abstimmender nicht Parteimitglied sein sollte. $\$ 265$ a StGB stellt das Erschleichen des Zutritts zu einer Veranstaltung in der Absicht, das Entgelt nicht zu entrichten, unter Strafe. Entsprechend dem Schutzzweck der Vorschrift sind nur solche Veranstaltungen und Einrichtungen gemeint, für deren Besuch oder Inanspruchnahme als wirtschaftliche Gegenleistung ein Entgelt verlangt wird. ${ }^{11}$

Das Entgelt für die Teilnahme an der Wahlversammlung einer Partei ist nicht in den Mitgliedsbeiträgen zu sehen. Zwar kann nach $\$ 10$ Abs. 2 Satz 2 ParteiG das Stimmrecht eines Mitglieds von der Erfüllung seiner Beitragspflicht abhängig gemacht werden; die Mit-

11 Vgl. Walter Perron, in: Adolf Schönke / Horst Schröder, Strafgesetzbuch - Kommentar, München 2010, $\$ 265$ a Rdnr. 2 . 
gliedsbeiträge einer Partei sind jedoch nicht als Entgelt im Sinne des $\$ 265$ a StGB zu verstehen. Dies wäre dann der Fall, wenn die Teilnahme an einem Parteitag eine wirtschaftliche Leistung der Parteien gegenüber ihren Mitgliedern darstellte.

Bei einem Parteitag erlangen die Mitglieder/Delegierten keine wirtschaftliche Leistung. Die Kosten werden in den Rechenschaftsberichten der Parteien der politischen Arbeit zugerechnet $^{12}$, da bei einem Parteitag politische Willensbildung betrieben und nicht eine wirtschaftliche Leistung der Partei an die Mitglieder/Delegierten weitergegeben wird. Schließlich ist der Parteitag eine wesentliche Ausprägung der politischen Willensbildung durch die Parteien. ${ }^{13}$

\subsection{Manipulation beim Wahlvorgang}

Denkbar ist eine strafrechtlich relevante Manipulation bei einem innerparteilichen Wahlvorgang durch Austausch, Veränderung, Hinzufügung oder Unterdrückung von Stimmzetteln oder sogar ganzen Urnen.

\section{(1) Veränderung und Hinzufügung von Stimmzetteln}

Bei der Manipulation von Vorstands- beziehungsweise Delegiertenwahlen durch den Austausch von Stimmzetteln, deren Veränderung (insbesondere bei Gruppenwahlen) sowie durch Hinzufügen nachgemachter oder erschlichener Stimmzettel könnte eine Urkundenfälschung nach $\$ 267$ StGB begangen werden.

Bei einem einzelnen Stimmzettel handelt es sich nicht um eine Urkunde im Sinne des $\$ 267$ StGB, da Stimmzettel den Aussteller nicht erkennen lassen. ${ }^{14}$ Die Rechtsprechung geht aber von der Entstehung einer fälschungsfähigen Gesamturkunde ${ }^{15}$ aus, die aus sämtlichen von den Wählern abgegebenen Stimmzettel in ihrer Gesamtheit innerhalb der Wahlurne zusammen mit der Wählerliste je Wahlbezirk gebildet wird. ${ }^{16}$ Der BGH begründet seine Auffassung damit, dass vom Erfordernis der Bestimmtheit oder Bestimmbarkeit des Ausstellers ausnahmsweise abzusehen sei, wenn der Gesetzgeber selbst verlange, dass im öffentlichen Interesse Name und Person der Aussteller der einzelnen Wahlzettel verborgen blieben. ${ }^{17}$ Für die Frage, ob diese Rechtsprechung auch auf parteiinterne Wahlen über-

12 Siehe die Bekanntmachung von Rechenschaftsberichten politischer Parteien für das Kalenderjahr 2008; BT-Drs. 17/630; BT-Drs. 17/870; BT-Drs. 17/2460.

13 Gleiches gilt auch für die Teilnahme an Aufstellungsversammlungen. Bei der Kandidatenaufstellung üben die Mitglieder ihre originären Rechte aus. Das Stimmrecht ist bei Aufstellungsversammlungen ohnehin nicht an die Beitragszahlung geknüpft, da die Bestimmung des $\$ 10$ Abs. 2 Satz 2 ParteiG eng ausgelegt und in seinem Geltungsbereich lediglich auf parteiinterne Abstimmungen beschränkt wird. So kann ein Mitglied trotz mehriähriger Zahlungsrückstände nicht von einer Aufstellungsversammlung ausgeschlossen werden. Vgl. Wolfgang Schreiber, a.a.O. (Fn. 5), $\$ 21$ Rdnr. 12.

14 Vgl. BGHSt 13, S. 108 ff., S. 112; OLG Koblenz, NStZ 1992, S. 134 f.

15 Vgl. Dominik Härtel, a.a.O. (Fn. 2), S. 155 f. spricht von einer „zusammengesetzten Urkunde“.

16 Vgl. BGH, a.a.O. (Fn. 14); kritisch Ingeborg Puppe, Die neue Rechtsprechung zu den Fälschungsdelikten, in: JZ 1997, S. 490 - 500, S. 490; Dominik Härtel, a.a.O. (Fn. 2).

17 Vgl. BGH, a.a.O. (Fn. 14). 
tragen werden kann, ist es erforderlich, sich den Ablauf einer Wahl innerhalb einer Partei zu vergegenwärtigen.

In der Regel füllen die Mitglieder beziehungsweise Delegierten die Stimmzettel an ihren Sitzplätzen oder in den bereit stehenden Wahlkabinen aus. Eingesammelt werden die Stimmzettel von Wahlhelfern, die mit Wahlurnen durch die Reihen gehen oder an den Wahlkabinen positioniert sind. Hier zeigt sich ein erster entscheidender Unterschied zum Ablauf der Wahlen an offiziellen Wahltagen in den Wahllokalen: Während es dort lediglich eine Wahlurne gibt, sind bei Parteiwahlen in aller Regel mehrere im Einsatz. Eine Zusammenführung aller Stimmzettel findet erst bei der Auszählung statt.

Ein zweiter Unterschied besteht darin, dass die Anwesenheitsliste nicht als Nachweis für die Anzahl der abgegeben Stimmen taugt. Es ist bei Parteien nicht ungewöhnlich, dass sich ein stimmberechtigtes Mitglied trotz Anwesenheit an der Versammlung nicht an der Abstimmung beteiligt. Im Gegensatz dazu sind an einem Wahltag in einem Wahllokal nur die Stimmzettel der Personen in einer Wahlurne, die auch das Wahllokal aufgesucht haben und deren Stimmabgabe von den Wahlhelfern vermerkt wurde.

Bei einer öffentlichen Wahl ist somit mittels des Wählerverzeichnisses nachvollziehbar, wer von seinem Wahlrecht Gebrauch gemacht hat. Gerade dies ist aber bei einer parteiinternen Wahl nicht der Fall. Bei dieser lässt sich der Kreis der Personen, die die Möglichkeit hatten, einen Stimmzettel abzugeben, zwar anhand der Anwesenheitsliste eingrenzen, aber nicht feststellen, wer sein Wahlrecht tatsächlich ausgeübt hat. Eine „Bestimmbarkeit des Ausstellers" der Stimmzettel im Zusammenhang mit der Anwesenheitsliste ist damit nicht gegeben. Folglich kann bei Parteiwahlen mangels Bestimmbarkeit des Ausstellerkreises die Entstehung einer Gesamturkunde oder einer zusammengesetzten Urkunde nicht angenommen werden, damit auch keine Urkundenfälschung nach $₫ 267$ StGB begangen werden. Also ist Manipulation bei einem innerparteilichen Wahlvorgang durch Austausch beziehungsweise Unterdrücken von Stimmzetteln oder sogar ganzen Urnen nicht nach $\$ 267$ StGB strafbewehrt. Andere Normen, die ein solches Verhalten strafrechtlich sanktionieren könnten, sind nicht ersichtlich, Vermögensdelikte sind mangels eines finanziellen Schadens nicht einschlägig. Daher bleibt die Manipulation an Stimmzetteln und Urnen bei Vorstands- und Delegiertenwahlen straflos.

\section{(2) Unterdrücken von Stimmzetteln}

Hinsichtlich des Unterdrückens von Stimmzetteln (als Folge „falscher“ Auszählung) dürfte in der Regel ein strafrechtlich relevanter Diebstahl nach $\$ 242$ StGB vorliegen. Mit der Wegnahme von Stimmzetteln wird eine fremde bewegliche Sache einem anderen rechtswidrig mit Aneignungsabsicht entwendet. Gewahrsam an den Stimmzetteln hat die jeweilige Parteigliederung, vertreten durch ihren Vorstand. Dass je nach Einzelfall die Stimmenauszähler noch Mitgewahrsam haben, steht einer Tatbestandsverwirklichung ebenso nicht entgegen wie die Tatsache, dass die unterdrückten Stimmzettel vernichtet werden. Schließlich werden durch die Vernichtung die ausgefüllten Stimmzettel der Parteigliederung rechtswidrig zur Auswertung und Aufbewahrung entzogen.

Eine Strafbarkeit wegen Unterschlagung nach $₫ 266$ StGB scheidet aus, da durch das Unterdrücken von Stimmzetteln keine Vermögensschädigung eintritt. 


\section{Aufstellungsverfahren}

Die obigen Ausführungen gelten hinsichtlich der Strafbarkeit bei Manipulationen im Rahmen von Aufstellungsverfahren genauso. Darüber hinaus ist die Frage zu klären, ob neben den Urkundendelikten und der möglichen Strafbarkeit nach $\$ 156$ StGB in Verbindung mit $₫ 21$ Abs. 6 BWahlG (beziehungsweise $\$ 27$ Abs. 5 BWahlG für die Aufstellung von Landeslisten) auch die Vorschriften über die Wahlstraftaten nach $\$ \$ 107$ ff. StGB Anwendung finden können.

\subsection{Strafbarkeit nach $₫ 156$ StGB in Verbindung mit $₫ 21$ Abs. 6 BWahlG}

An Eides statt wird nach $\$ 21$ Abs. 6 BWahlG versichert, dass die Mitglieder- beziehungsweise Delegiertenversammlung in geheimer Abstimmung einen Bewerber benannt hat, jeder stimmberechtigte Teilnehmer der Versammlung abstimmungsberechtigt war und die Bewerber Gelegenheit hatten, sich und ihr Programm der Versammlung in angemessener Zeit vorzustellen. Der jeweils zuständige Wahlleiter ist zur Abnahme einer solchen Versicherung an Eides statt zuständig; $\$ 21$ Abs. 6 Satz 3 BWahlG sieht vor, dass er als Behörde im Sinne des $\$ 156$ StGB gilt.

Der Umfang der Versicherung wird in den Anlagen 17 und 18 zu $\$ 34$ Abs. 5 Nr. 3 lit. a BWahlO (Kreiswahlvorschläge) sowie den Anlagen 23 und $24 \mathrm{zu} \$ 39$ Abs. 4 Nr. 1 und Nr. 3 BWahlO (Landesliste) konkretisiert. Deren Inhalt umfasst die sich aus dem Grundgesetz ergebenden, zwingend einzuhaltenden Wahlrechtsgrundsätze der Allgemeinheit, der Unmittelbarkeit, der Freiheit, der Gleichheit der Wahl sowie das Wahlgeheimnis, angepasst an die Besonderheiten einer Aufstellungsversammlung. ${ }^{18}$ Neben dem Versammlungsleiter geben zwei weitere, zu Beginn der jeweiligen Versammlung gewählte Personen die Versicherung an Eides statt ab.

Ein wichtiger Grund für diese Regelung ist, dass den Stellungnahmen der Versicherer an Eides statt aufgrund der Strafbewehrtheit ihrer Aussagen und der korrespondierenden Pflicht zur Überwachung der Versammlung ein erhöhter Beweiswert zukommt. Dies ist dann von Bedeutung, falls es im Rahmen der Zulassungsentscheidung erforderlich sein sollte, dass der zuständige Wahlausschuss eine Prüfung des Ablaufs einer Aufstellungsversammlung vornimmt. ${ }^{19}$ Mögliche Mängel bei einer Aufstellungsversammlung müssen somit, um zu einer Prüfung durch den Wahlleiter beziehungsweise die Wahlausschüsse zu führen, substantiiert vorgebracht werden.

Den Versicherern an Eides statt kommt aufgrund des von der Versicherung abgedeckten Umfangs eine besonders verantwortungsvolle Stellung in der Aufstellungsversammlung zu, beginnend mit der Kontrolle der Ausgabe der Wahlunterlagen bis hin zur Auszählung, da ebenso das Abstimmungsergebnis von der eidesstattlichen Versicherung umfasst ist.

18 Vgl. BVerfGE 89, S. 243 ff., S. 251; Il Sun Hong, a.a.O. (Fn. 6), S. 58; Wolfgang Schreiber, a.a.O. (Fn. 5), \$ 21 Rdnr. 43 f. So ist es nicht zwingend erforderlich, dass jeder Wähler seine Stimme tatsächlich geheim abgibt, es muss lediglich die faktische Möglichkeit dazu bestehen, um den Grundsatz der geheimen Wahl einzuhalten.

19 Vgl. BVerfGE 89, S. 243 ff., S. 254; Wolfgang Schreiber, a.a.O. (Fn. 5), \$26 Rdnr. 2, 17. 
Sie müssen den gesamten Ablauf überwachen, etwaige Fehler noch im Laufe der Wahlversammlung verhindern, abstellen und unterbinden. Somit sind die Versicherer an Eides statt verpflichtet, Anhaltspunkten für etwaige Unregelmäßigkeiten im Ablauf nachzugehen, wollen sie keine falsche Versicherung an Eides statt abgeben. Schließlich ist gemäß $\$ 161$ StGB auch schon die fahrlässige Versicherung an Eides statt strafbar; der bedingte Vorsatz für die Verwirklichung des subjektiven Tatbestands des $\$ 156$ StGB reicht aus. ${ }^{20}$

Dies stellt hohe Anforderungen an die Kenntnisse dieser Personen über den Ablauf einer solchen Veranstaltung sowie die rechtlichen Hintergründe des Wahlrechts, schließlich führt nicht jeder Fehler zwingend zu einem relevanten Wahlfehler. ${ }^{21}$ Hier sind viele Fallstricke zu finden, angefangen von der Bestimmung des Kreises der wahlberechtigten Personen über die Gestaltung der Stimmzettel und der benutzten Stifte sowie den Aufbau der Wahlkabinen bis zum Zustand der Wahlurnen und dem Auszählvorgang. Dem Leiter der Versammlung kommt bei der Durchführung der Versammlung daher eine besonders herausgehobene Bedeutung zu. Es kann nicht zwingend darauf vertraut werden, dass immer alles im Detail perfekt vorbereitet ist, so dass sich keine Fehler einschleichen können. ${ }^{22}$

Sollten bei der Manipulation des Ausgangs eines Aufstellungsverfahrens die Täter mit den Versicherern an Eides statt nicht identisch sein, könnte $₫ 160$ Abs. 1 Alt. 2 StGB zur Anwendung kommen, der die Verleitung zur Falschaussage unter Strafe stellt. Verleitung bedeutet die Einwirkung auf einen anderen, die eidesstattliche Versicherung abzugeben, die dieser - wenn auch fahrlässig - für richtig hält. Dies kann sowohl durch Täuschung als auch durch Ausnutzung eines Irrtums geschehen. ${ }^{23}$

\subsection{Strafbarkeit nach $₫ 107 \mathrm{~b}$ Abs. 1 Nr. 4 StGB}

Aus dem Bereich der Wahlstraftaten ist die Bestimmung des $\$ 107 \mathrm{~b}$ Abs. 1 Nr. 4 StGB auch auf parteiinterne Wahlen anwendbar. Danach macht sich strafbar, wer sich als Bewerber für eine Wahl aufstellen lässt, obwohl er nicht wählbar ist, sofern die Tat nicht in anderen Vorschriften mit schwererer Strafe bedroht ist. ${ }^{24}$

$20 \mathrm{Vgl}$. Theodor Lenckner, in: Adolf Schönke / Horst Schröder, a.a.O. (Fn. 11), \$156 Rdnr. 34.

21 Vgl. BVerfGE 89, S. 243 ff., S. 253; Wolfgang Schreiber, a.a.O. (Fn. 5), \$26 Rdnr. 20; Jörn Ipsen, in: ders. (Hrsg.), Parteiengesetz, München 2008, $\$ 17$ Rdnr. 12.

22 So musste beispielsweise die FDP die Aufstellung ihrer Bundesliste zur Europawahl 2004 wegen eines Verfahrensfehlers wiederholen, weil das Präsidium unter Berufung auf die Parteisatzung einer Person verwehrt hatte, sich selbst als Kandidat vorzuschlagen. Die Satzungsregelung steht jedoch im Widerspruch zum EuWahlG. Vgl. „FDP muss Europaparteitag wiederholen“, in: Spiegel Online vom 8. März 2004, http://www.spiegel.de/politik/deutschland/0,1518,289608,00.html; „FDP muss Europaparteitag wiederholen“, in: sueddeutsche.de vom 8. März 2004, http://www. sueddeutsche.de/politik/nach-klage-fdp-muss-europaparteitag-wiederholen-1.307923 (Abruf jeweils am 25. September 2011); Melanie Werner, Gesetzesrecht und Satzungsrecht bei der Kandidatenaufstellung politischer Parteien, Osnabrück 2010, S. $138 \mathrm{ff}$.

23 Vgl. Theodor Lenckner / Nikolaus Bosch, in: Adolf Schönke / Horst Schröder, a.a.O. (Fn. 11), \$160 Rdnr. 7; Thomas Fischer, Strafgesetzbuch und Nebengesetze, München 2009, $\$ 160$ Rdnr. 3.

24 Korrespondierend hierzu heißt es in $\$ 14$ WahlprüfungsG, dass auch nach Ablauf der Einspruchsfrist von zwei Monaten ( $\$ 2$ Abs. 4 WahlprüfungsG) gegen eine Wahl der Präsident des Bundestages Einspruch gegen die Gültigkeit einer Wahl einlegen kann, wenn sich Zweifel ergeben, ob ein Abgeordneter zum Zeitpunkt der Wahl wählbar war. 
Nach ihrem Wortlaut wird die Wahlbehinderung verwirklicht, wenn sich eine Person von einer Partei als Bewerber zu einer Wahl aufstellen lässt, obwohl ihm beispielsweise das aktive Wahlrecht aberkannt wurde oder er - außer bei Kommunal- oder Europawahlen - kein deutscher Staatsbürger ist. Die Bestimmung ist auf von einer Partei gewählte Bewerber anwendbar, denn die Vorschrift spricht von „aufstellen“. Dieser Begriff findet im Wahlrecht sowie im Parteiengesetz auf die „Benennung“ von Bewerbern durch Parteien Anwendung. ${ }^{25}$

$\mathrm{Zu}$ fragen ist allerdings, wann der Tatbestand vollendet wird. Dominik Härtel sieht vor dem Hintergrund des Schutzzweckes des staatlichen Wahlvorbereitungsapparates die Entscheidung über die Zulassung der Wahlvorschläge nach $\$ \$ 26,28$ BWahlG als maßgeblichen Vollendungszeitpunkt an. ${ }^{26}$ Er legt die Norm nicht im Sinne des $\$ 21$ Abs. 1 Satz BWahlG aus, da das StGB beim Vorhandensein mehrerer Möglichkeiten der Kandidatenaufstellung sich nicht auf die Form der Aufstellung in etablierten Parteien beschränken will.

Dem ist jedoch entgegenzuhalten, dass es nach dem Wortlaut der Norm nicht darauf ankommen kann, ob der nach dem Wahlrecht zuständige Vorstand einer Partei den Wahlvorschlag bei dem zuständigen Wahlleiter einreicht oder die nach Parteisatzung vorgesehene Stelle gemäß $\$ 21$ Abs. 4 BWahlG Einspruch gegen die Aufstellung eines Bewerbers erhebt. Neben dem deutlichen Bezug der Norm auf den Wortlaut des $\$ 21$ Abs. 1 Satz 1 BWahlG ist zu bedenken, dass zu den beim Wahlleiter einzureichenden Dokumenten auch eine von der Gemeindebehörde des Wohnorts auszustellende Bescheinigung der Wählbarkeit des Bewerbers gehört. ${ }^{27}$ Spätestens bei der Beibringung dieser Bescheinigung würde die fehlende passive Wahllegitimation auffallen. Stellte man daher auf den Zeitpunkt der $\mathrm{Zu}$ lassung des Wahlvorschlags durch die zuständigen Wahlausschüsse ab, würde die Strafrechtsvorschrift in ihrem Sinngehalt ausgehöhlt, eine Verwirklichung wäre nur im Fall der Fälschung der Bescheinigung über die Wählbarkeit möglich. Bei der Aufstellung eines Bewerbers durch eine Partei ist daher als Vollendungszeitpunkt für die Strafbarkeit auf die Verkündung des Wahlergebnisses durch den Versammlungsleiter abzustellen.

Da eine Versuchsstrafbarkeit nicht vorgesehen ist, bleibt eine Abstimmungsniederlage eines Nicht-Wahlberechtigten bei einer erfolgreichen Gegenkandidatur straffrei.

\subsection{Strafbarkeit nach den $\$ \$ 107 \mathrm{ff}$. StGB}

Wie gerade aufgezeigt, sind Handlungen, die zeitlich vor dem eigentlichen Wahlakt liegen, grundsätzlich geeignet, das Wahlergebnis zu verfälschen, und dementsprechend gemäß $\$ \$$ $107 \mathrm{ff}$. StGB strafrechtlich sanktioniert.

25 Auch Einzelbewerber können sich nach 107b Abs. 1 Nr. 4 StGB strafbar machen, sei es, dass sie als Einzelbewerber zur Wahl antreten oder von Wählergruppen/-vereinigungen vorgeschlagen werden. Dies ergibt sich aus dem Zusammenspiel mit $\$ 108 \mathrm{~d}$ Satz 2 StGB, in dem es heißt, dass einer Wahl oder Abstimmung das Unterschreiben eines Wahlvorschlags gleich steht. Hier wird der Bürger bei der Abgabe seiner Unterschrift darüber getäuscht, dass er damit erreichen könnte, dass ein Einzelbewerber zur Wahl zugelassen wird.

26 Vgl. Dominik Härtel, a.a.O. (Fn. 2), S. 86.

27 Siehe Anlage 16 zu $\$ 34$ Abs. 5 Nr. 2 und $\$ 39$ Abs. 4 Nr. 2 sowie Anlage 21 zu $\$ 39$ Abs. 3 BWahlO. Sofern keine Anhaltspunkte im Vorfeld der Aufstellungsversammlung vorliegen oder es sich um einen spontanen Wahlvorschlag handelt, wird diese Bescheinigung in der Regel erst nach einer erfolgreichen Benennung des Bewerbers durch eine politische Partei angefordert. 
Anwendbarkeit der $\$ \$ 107$ ff. StGB auf die Kandidatenaufstellung

Es ist nun die Frage zu beantworten, ob auch die übrigen Vorschriften über den Schutz von Wahlen und Abstimmungen des StGB, die das Vertrauen in öffentliche Wahlen schützen, also die Wahlbehinderung $(\$ 107)$, die Wahlfälschung (\$107a), die Fälschung von Wahlunterlagen (\$107b Abs. 1 Nr. 1-3), die Verletzung des Wahlgeheimnisses (\$107c), die Wählernötigung (\$ 108), die Wählertäuschung (\$ 108a) und die Wählerbestechung ( $\$ 108$ b), Anwendung auf Kandidatenaufstellungen finden, die ja ebenfalls vor dem eigentlichen Wahlakt stattfinden.

\section{(1) Begriff der Wahl in $\$ 108 \mathrm{~d}$ StGB}

In $\$ 108 \mathrm{~d}$ StGB, der den Anwendungsbereich der $\$ \$ 107 \mathrm{ff}$. StGB definiert, heißt es, dass die $\$ \$ 107$ bis 108d StGB für Wahlen zu den Volksvertretungen, für die Wahl der Abgeordneten des Europäischen Parlaments, für sonstige Wahlen und Abstimmungen des Volkes im Bund, in den Ländern, Gemeinden und Gemeindeverbänden sowie für Urwahlen in der Sozialversicherung gelten. Einer Wahl oder Abstimmung steht das Unterschreiben eines Wahlvorschlags oder das Unterschreiben für ein Volksbegehren gleich.

Mit der Bestimmung des $\$ 108 \mathrm{~d}$ StGB hat der Gesetzgeber eine Regelung geschaffen, die insbesondere für die $\$ \$ 107 \mathrm{ff}$. StGB verbindlich ist. Er hat damit zum Ausdruck gebracht, dass sich der strafrechtliche Schutz nur auf Wahlen und Abstimmungen des aufgezählten Bereichs erstrecken soll. ${ }^{28}$ Eine Legaldefinition des Begriffs der Wahl ist damit jedoch nicht verbunden. ${ }^{29}$ Dass es sich hierbei um einen staatsrechtlichen Begriff handelt, der ins Strafrecht eingeführt wurde, ändert nichts daran, dass er auch weiter vom Staatsrecht zu definieren ist; $\$ 108 \mathrm{~d}$ StGB bezweckt lediglich eine Beschränkung des Schutzbereichs. ${ }^{30}$

Wahlen im Sinne der $\$ \$ 107$ ff. StGB sind nach $\$ 108 d$ StGB grundsätzlich nur Volkswahlen und -abstimmungen, das heißt Stimmabgaben des Volkes in Ausübung staatsbürgerlicher Rechte. ${ }^{31}$ Damit schützt der Gesetzgeber ausschließlich die Akte der Willensbildung, die den Erfordernissen der allgemeinen verfassungsrechtlichen Wahlrechtsgrundsätze der Art. 38 Abs. 1 Satz 1, 28 Abs. 1 Satz 2 GG genügen, also allgemeine, unmittelbare, freie und geheime Wahlen. ${ }^{32}$

Entscheidend für die Anwendbarkeit der $\$ \$ 107$ ff. StGB auf parteiinterne Kandidatenaufstellungen ist also die Frage, ob Aufstellungen bereits als Bestandteil der öffentlichen

28 Vgl. Georg Bauer / Duscha Gmel, in: Heinrich Wilhelm Laufhütte / Ruth Rissing-van-Saan / Klaus Tiedemann (Hrsg.), Leipziger Kommentar Strafgesetzbuch, Berlin 2007, Vor $\$ \$ 107$ Rdnr. 3; Henning Ernst Müller, in: Wolfgang Joecks / Klaus Miedebach (Hrsg.), Münchener Kommentar zum Strafgesetzbuch, München 2005, $\$ 108 d$ Rdnr. 2; Hans-Joachim Rudolphi, in: ders. I Jürgen Wolters (Hrsg.), Systematischer Kommentar zum Strafgesetzbuch, Köln 2001, § 108d Rdnr. 1.

29 So aber Albin Eser, in: Adolf Schönke / Horst Schröder, a.a.O. (Fn. 11), \$108d Rdnr. 1; Bodo Vogler / Henning Rosenau, in: Helmut Satzger / Bertram Schmitt / Gunter Widmaier (Hrsg.), Strafgesetzbuch, Köln 2009, \$108d Rdnr. 1.

30 Vgl. Gerhard Wolf, a.a.O. (Fn. 2), S. 27. Das BVerfG (NVwZ 1993, S. 55 ff., S. 55) hat dazu ausgeführt, dass „die Bestimmtheit des Straftatbestandes nicht dadurch in Frage gestellt (wird), dass seine Anwendung die Beurteilung von wahlrechtlichen Vorfragen (...) erfordert, die nicht im StGB, sondern in den Vorschriften des maßgeblichen Wahlrechts (...) geregelt sind.“

31 Vgl. Albin Eser, a.a.O. (Fn. 29), $\$ 108$ d Rdnr. 2; Hans-Joachim Rudolphi, a.a.O. (Fn. 28), $\$ 108 d$ Rdnr. 1.

32 Vgl. Gerhard Wolf, a.a.O. (Fn. 2), S. 29. 
Wahlen anzusehen sind oder es sich dabei lediglich um reine Vorbereitungsakte handelt, die damit aus dem Anwendungsbereich des $₫ 108 \mathrm{~d}$ StGB fallen.

\section{(2) Rechtliche Grundlagen der Kandidatenaufstellung}

$\$ 17$ PartG stellt fest, dass die Aufstellung von Bewerbern für Wahlen zu Volksvertretungen von den Wahlgesetzen und den Satzungen der Parteien geregelt wird. Hier überschneidet sich also die innere Ordnung der Parteien mit dem staatlichen Wahlrecht. ${ }^{33}$ Einerseits gehört die Kandidatenaufstellung zum Parteienrecht, denn schließlich sind die Parteien Veranstalter. Dem Sachzusammenhang nach und bei einer schwerpunktmäßigen Verortung steht sie aber dem Wahlrecht näher. ${ }^{34}$ Dies ergibt sich aus dem Umfang der Regelungskompetenzen der Parteien in ihren Satzungen hinsichtlich der Kandidatenaufstellung.

Grundgesetz, Wahlgesetz und Wahlordnung liefern detaillierte wahlrechtliche Maßstäbe für die Kandidatenauswahl. Ihr Ausgangspunkt ist $\$ 21 \mathrm{BWahlG}$, der die wahlgesetzlichen Anforderungen maßgeblich normiert. In $₫ 21$ Abs. 1 BWahlG ist über die örtliche Abgrenzung die Passivlegitimation der wahlberechtigten Parteimitglieder vorgegeben. Absatz 3 der Vorschrift steckt den Rahmen für die Aufstellung ab; es wird geheim gewählt, jeder Teilnehmer ist vorschlagsberechtigt, den Bewerbern ist eine angemessene Zeit einzuräumen, sich und ihr Programm vorzustellen. Zudem wird in Abhängigkeit von der Wahlperiode die Zeitspanne vorgegeben, in der eine Aufstellung durchzuführen ist. Über die Anlagen zur BWahlO wird überdies der Inhalt des Protokolls vorgeschrieben, somit auch de facto die Tagesordnung einer Aufstellungsversammlung. Damit sind der genaue Ablauf sowie die konkrete Durchführung einer Kandidatenaufstellung bestimmt. Des Weiteren müssen die Parteien bei der Bewerberaufstellung die Mindestregeln einer demokratischen Wahl einhalten, die sich aus Art. 21 Abs. 1 Satz 3, 38 Abs. 1 Satz 1 GG ergeben. ${ }^{35}$ Aus diesen Verfassungsgrundsätzen leiten sich weitere konkrete und enge Vorgaben für die Durchführung der Aufstellungsversammlung ab.

Der den Parteiensatzungen vorbehaltene Spielraum zur Ausgestaltung des Aufstellungsverfahrens ist im Vergleich dazu gering. ${ }^{36}$ Die wesentlichen Punkte bestehen darin, dass die Parteien entscheiden können, ob die Bewerberaufstellung als Mitglieder- oder Delegiertenversammlung durchgeführt wird. Ferner wird ihnen zugestanden, Regelungen zur Beschlussfähigkeit und den Mehrheitsverhältnissen zu treffen. ${ }^{37}$ Zudem kann satzungs-

33 Vgl. BVerfGE 89, S. 243 ff., S. 252; Jörn Ipsen, a.a.O. (Fn. 21), \$ 17 Rdnr. 1; Wolfgang Schreiber, a.a.O. (Fn. 5), Rdnr. 15. Wilhelm Henke (Das Recht der politischen Parteien, Göttingen 1972, S. 146 f.) vertritt die Ansicht, dass die Vorschriften über die Kandidatenaufstellung in den Zusammenhang der demokratischen inneren Ordnung einer Partei gehören, das Wahlrecht hingegen ein staatliches Verfahren regelt. Zur Frage der verfassungsrechtlichen Einordnung der Kandidatenaufstellung vgl. Il Sun Hong, a.a.O. (Fn. 6), S. 35 ff.

34 Vgl. Jörn Ipsen, a.a.O. (Fn. 21), $\$ 17$ Rdnr. 1; Wolfgang Schreiber, a.a.O. (Fn. 5), $\$ 21$ Rdnr. 5; Melanie Werner, a.a.O. (Fn. 22), S. 55 ff., S. 68.

35 Vgl. BVerfGE 41, S. 399 ff., S. 417; BVerfGE 47, S. 253 ff., S. 282; BVerfGE 89, S. 243 ff., S. 252 f.; Wolfgang Schreiber, a.a.O. (Fn. 5), \$21 Rdnr. 1.

36 Dennoch besteht somit ein Kernbereich parteiautonomer Willensbildung, der durch verfassungsrechtliche Schranken nicht verletzt werden darf, damit die Parteien vor unverhältnismäßiger staatlicher Einflussnahme geschützt werden. Vgl. SächsVerfGH, LKV 2006, S. 270 ff., S. 270 f.

37 Zur verfassungsrechtlichen Zulässigkeit der möglichen Wahlverfahren vgl. Il Sun Hong, a.a.O. (Fn. 6), S. $64 \mathrm{ff}$. 
rechtlich geregelt werden, welche Voraussetzungen an die Bewerber gestellt werden, beispielsweise ob sie Parteimitglied sein oder eine bestimmte Zeit im Wahlkreis wohnhaft sein müssen. ${ }^{38}$ Bei Listenaufstellungen haben inzwischen nahezu alle Parteien eine Quotenregelung zugunsten der Frauenförderung erlassen. ${ }^{39}$ Ansonsten verbleibt den Parteien noch die Regelung über die Ladungsfristen, den genauen Zeitpunkt und den Ort der Versammlung.

\section{(3) Die Kandidatenaufstellung im Wahlprüfverfahren}

Ein Blick auf die Konsequenzen möglicher Fehler bei der Kandidatenaufstellung kann bei der Klärung der Frage, ob diese bereits Teil der gesetzlich geschützten Wahlvorbereitung ist, behilflich sein.

Der Rechtsschutz gegen Maßnahmen der Kandidatenaufstellung wird von der Verfassung bestimmt. Art. 41 GG, nach dem die Wahlprüfung eine Sache des Bundestages ist, gegen dessen Entscheidung die Beschwerde an das BVerfG zulässig ist, stellt insoweit eine Sonderregelung dar. ${ }^{40}$ Rechtsgrundlage für das Wahlprüfungsverfahren ist $₫ 49$ BWahlG, der unmittelbar Ausfluss des Art. 41 GG ist. In $\$ 49$ BWahlG heißt es, dass Entscheidungen und Maßnahmen, die sich unmittelbar auf das Wahlverfahren beziehen, nur mit den in diesem Gesetz und in der Bundeswahlordnung vorgesehenen Rechtsbehelfen sowie im Wahlprüfungsverfahren nach dem Wahlprüfungsgesetz angefochten werden können.

Gegenstand der Wahlprüfung ist danach die Gesamtheit der Wahlvorgänge, das heißt von ihrer organisatorischen Vorbereitung, dem Wahlakt selber und der Ermittlung und Feststellung der endgültigen Ergebnisse bis einschließlich der Verteilung der Sitze. ${ }^{41}$ Prüfungsmaßstab bilden dabei alle Rechtssätze, die anlässlich der Wahl zur Anwendung kommen. ${ }^{42}$

Hiervon sind auch die Rechtssätze über die parteiinternen Aufstellungsverfahren umfasst. ${ }^{43}$ Das BVerfG hat dazu ausgeführt, dass in einem Wahlprüfungsverfahren der Rüge von Mängeln bei der Anwendung der für die Wahl geltenden wahlrechtlichen Bestimmungen nachgegangen wird. Weiter heißt es in der Entscheidung: „Solche Wahlfehler können nicht nur von amtlichen Wahlorganen begangen werden, sondern auch von Dritten, soweit sie unter Bindung an wahlgesetzliche Anforderungen kraft Gesetzes Aufgaben bei der Organisation einer Wahl erfüllen. (...) bei der Wahl gibt es nach dem geltenden Bundestagswahlrecht für den Wahlberechtigten keine Möglichkeit, andere als vorgeschlagene Bewerber zu wählen oder mit der Zweitstimme auch nur Einfluss auf die Listenplätze der Kandidaten zu nehmen. (...) Wird bei den Aufstellungsverfahren der Kernbestand an Verfahrensgrundsätzen, der sich aus dem Verfassungsrecht und den Wahlgesetzen ergibt, nicht eingehalten,

38 Vgl. Jörn Ipsen, a.a.O. (Fn. 21), \$17 Rdnr. 17; Wolfgang Schreiber, a.a.O. (Fn. 5), \$21 Rdnr. 25; Christian Seiler, Referendarklausur - Öffentliches Recht: Streit in der Partei, in: JuS 2005, S. $1107-1112$, S. 1110.

39 Vgl. Ingwer Ebsen, Verbindliche Quotenregelungen für Frauen und Männer in Parteistatuten, Heidelberg 1988; Jörn Ipsen, a.a.O. (Fn. 21), \$17 Rdnr. 17 ff.; Il Sun Hong, a.a.O. (Fn. 6), S. 78 ff.; Melanie Werner, a.a.O. (Fn. 22), S. 74 ff. (CDU), S. 96 ff. (CSU), S. 101 ff. (SPD), S. $121 \mathrm{ff.}$ (Bündnis 90/Die Grünen), S. 138 ff. (FDP), S. 148 ff. (Die Linke), S. 191 ff. (zur Vereinbarkeit mit der Verfassung).

40 Vgl. Wolfgang Schreiber, a.a.O. (Fn. 5), \$49 Rdnr. 2.

41 Vgl. ebenda, $\$ 49$ Rdnr. 1, 14; BVerfGE 1, S. 208 ff, S. 238; BVerfGE 40, S. 1 ff., S. 29; BVerfGE 89, S. 243 ff., S. 250 f.; Il Sun Hong, a.a.O. (Fn. 6), S. 97.

42 Vgl. BVerfGE 89, S. 243 ff., S. 250 f.; Wolfgang Schreiber, a.a.O. (Fn. 5), \$ 49 Rdnr. 1, 14.

43 Vgl. ebenda. 
so wird die demokratische Legitimationswirkung staatlicher Wahlen schlechthin in Frage gestellt und begründet die Gefahr der Verfälschung des demokratischen Charakters der Wahl bereits in ihrer Grundlage." 44

Den Sachverhalt, über den das BVerfG in diesem Fall zu entscheiden hatte, betraf einen Fall, in dem Fehler im parteiinternen Aufstellungsverfahren gerügt wurden. Im selben Jahr hatte das Hamburger Verfassungsgericht die Wahl eines ganzen Parlaments wegen Fehler bei der parteiinternen Kandidatenaufstellung für nichtig erklärt und damit eine Neuwahl erzwungen. ${ }^{45}$ Mit diesen Entscheidungen wurde bewusst die der Wahlvorbereitung dienende Kandidatenauswahl durch Parteien in das Wahlprüfungsverfahren einbezogen. ${ }^{46}$ Wahlrechtlich unterliegen die Parteien damit den gesetzlichen Bindungen, die der Gesetzgeber zur Sicherung des freien Wahlvorschlagsrechts der Stimmberechtigten normiert und für deren Einhaltung er eine Kontrolle geschaffen hat. ${ }^{47}$ Eine Verletzung der Vorschriften über die Kandidatenaufstellung ist in allen Phasen des Wahlverfahrens von Amts wegen zu prüfen. ${ }^{48}$

Bei der Kandidatenaufstellung ist die Beachtung der in den Wahlgesetzen enthaltenen Vorschriften wahlrechtlich verbindlich und erheblich, die Einhaltung der nach der Parteisatzung geltenden Bestimmungen führt jedoch nicht zwingend zu einem fehlerhaften Wahlvorschlag. ${ }^{49}$ Verstöße gegen die satzungsrechtlichen Verfahrensvorgaben oder rechtswidrige Satzungsbestimmungen führen nicht automatisch zu zurückzuweisenden Wahlvorschlägen.

Die Kontrolle der Einhaltung der Vorschriften bei der Bewerberaufstellung liegt dementsprechend nicht allein in den Händen der Parteivorstände oder der Parteischiedsgerichte, sondern entscheidend bei staatlichen Organen, nämlich dem Wahlprüfungsausschuss und den amtlichen Wahlleitern. ${ }^{50}$ Folglich spricht auch das Wahlprüfungsverfahren für eine Nähe des Aufstellungsverfahrens zum Wahlrecht.

(4) Kandidatenaufstellung als Bestandteil der Wahl

Vor diesem Hintergrund spricht bereits viel dafür, die Aufstellung von Kandidaten durch Parteien als geschützten Bestandteil der Wahl anzusehen. Bestätigt wird dies durch die Aus-

44 BVerfGE 89, S. 243 ff., LS 1 und 2, S. 251 ff.

45 Vgl. Hbg. VerfG, NVwZ 1993, S. 1083 ff. Die Entscheidung ist vielfach kritisiert worden, jedoch bezog sich die Kritik hauptsächlich auf die Begründung und nicht auf die Einbeziehung des Aufstellungsverfahrens in das Wahlprüfverfahren. Siehe mit weiteren Nachweisen Il Sun Hong, a.a.O. (Fn. 6), S. 18.

46 Vgl. BayVerfGH, NVwZ-RR 2010, S. 213 f., S. 213; Christian Seiler, a.a.O. (Fn. 38), S. 1109.

47 Vgl. BVerfGE 47, S. 253 ff., S. 283; BVerfGE 89, S. 243 ff., S. 251 f.

48 Vgl. BayVerfGH, a.a.O. (Fn. 46), S. 213.

49 Vgl. BVerfGE 89, S. 243 ff., LS 2b, S. 252.

50 Der Kreiswahlleiter hat nach $\$ 25$ Abs. 1 BWahlG die Aufgabe, die Kreiswahlvorschläge unverzüglich nach Eingang zu prüfen. Stellt er bei einem Kreiswahlvorschlag Mängel fest, so benachrichtigt er sofort die von der Partei für diesen Wahlvorschlag gewählte Vertrauensperson und fordert sie auf, behebbare Mängel rechtzeitig zu beseitigen. Nach $\$ 26$ Abs. 1 BWahlG entscheidet der Kreiswahlausschuss über die Zulassung der Kreiswahlvorschläge. Er hat Kreiswahlvorschläge zurückzuweisen, wenn sie verspätet eingereicht werden oder den Anforderungen nicht entsprechen, die durch dieses Gesetz und die Bundeswahlordnung aufgestellt sind, es sei denn, dass in diesen Vorschriften etwas anderes bestimmt ist. Identische Vorschriften finden sich in den $\$ \$ 27,28$ BWahlG über die Prüfung der Landeslisten. Zu den möglichen Rechtsmitteln gegen eine Kandidatenaufstellung im laufenden Verfahren vgl. Il Sun Hong, a.a.O. (Fn. 6), S. 85 ff. 
führungen des BVerfG in seiner bereits erwähnten Entscheidung vom 20. Oktober 1993. Dort heißt es: „Die Aufstellung der Wahlkreis- und Listenkandidaten durch die Parteien ist (...) ein wesentlicher Bereich der Wahlvorbereitung; hierdurch wird eine notwendige Voraussetzung für die Wahl selbst geschaffen und das aktive und passive Wahlrecht unmittelbar berührt." 51

Diese Aussage des BVerfG erklärt sich daraus, dass die Parteien bei der Organisation der Wahl unter Bindung der wahlgesetzlichen Vorschriften maßgeblich mitwirken; dies ist unmittelbarer Ausfluss ihres verfassungsrechtlichen Auftrags zur Mitwirkung an der politischen Willensbildung gemäß Art. 21 Abs. 1 GG. ${ }^{52}$ Schließlich gehen die Wahlgesetze von Kandidatenaufstellungen durch die Parteien aus, denn gemäß $\$ 1$ Abs. 2 BWahlG werden die Hälfte der Sitze im Deutschen Bundestag sowie die so genannten Überhangmandate über die Listen der Parteien vergeben. Zudem sind seit der zweiten Wahlperiode nur noch Kandidaten über Parteien in den Bundestag gelangt. Damit besteht faktisch ein Parteienmonopol für die Erlangung eines Bundestagsmandats. ${ }^{53}$ Durch die Aufstellung von Bewerbern in so genannten sicheren Wahlkreisen beziehungsweise auf sicheren Listenplätzen werden (mittlerweile allerdings immer weniger) Abgeordneten-Mandate praktisch im Vorfeld der eigentlichen Wahl vergeben. ${ }^{54}$ Die eigentliche Wahl beschränkt sich dann auf eine Artikulation des Vertrauens in die sachliche Leistungsfähigkeit und persönliche Integrität der von den Parteien aufgebotenen Personen. ${ }^{55}$ Parlamentswahlen ohne die Mitwirkung der Parteien sind im bestehenden Wahlrecht nicht denkbar. ${ }^{56}$

Somit ist festzuhalten, dass der Begriff der Wahl den gesamten Wahlvorgang umfasst, von der Erfassung der Wahlberechtigten über die Aufstellung der Bewerber bis zur Stimmabgabe und der Auszählung nebst Feststellung des Wahlergebnisses. ${ }^{57}$

\section{(5) Ergebnis}

Das deutsche Wahlsystem fußt auf den Bewerberaufstellungen durch die Parteien; ohne ihre Beteiligung der politischen Parteien ist eine geordnete Wahl kaum vorstellbar, sie ist ein wesentliches Element des einheitlichen Wahlverfahrens im Sinne des Grundgesetzes. ${ }^{58}$ Dies legt

51 BVerfGE 89, S. 243 ff., S. 251; BayVerfGH, a.a.O. (Fn. 46), S. 213.

52 Vgl. Il Sun Hong, a.a.O. (Fn. 6), S. 35 f.; Wolfgang Schreiber, a.a.O. (Fn. 5), \$ 21 Rdnr. 1.

53 Vgl. Hans Herbert von Arnim, Wahl ohne Auswahl. Die Parteien und nicht die Bürger bestimmen die Abgeordneten, in: ZRP 2004, S. 115 - 119, S. 116; Wolfgang Schreiber, a.a.O. (Fn. 5), \$21 Rdnr. 1.

54 Vgl. ebenda; Il Sun Hong, a.a.O. (Fn. 6), S. 17; Karl-Heinz Seifert, Die politischen Parteien im Recht der Bundesrepublik Deutschland, Köln u.a. 1975, S. 369. Suzanne S. Schüttemeyer und Roland Sturm (Der Kandidat - das (fast) unbekannte Wesen. Befunde und Überlegungen zur Aufstellung der Bewerber zum Deutschen Bundestag, in: ZParl, 36. Jg. (2005), H. 3, S. 539 553, S. 547 f.) kommen zu dem Ergebnis, dass bereits der Ausgang einer Aufstellungsversammlung vielfach zuvor entschieden ist. Bei der Bundestagswahl 2002 trat bei den kleineren Parteien in 80 Prozent der Fälle nur ein Kandidat an, bei der SPD gab es in 33,5 Prozent und bei der CDU in 43,0 Prozent der Fälle Kampfkandidaturen.

55 Vgl. Wolfgang Schreiber, a.a.O. (Fn. 5); Wilhelm Henke, a.a.O. (Fn. 33), S. 27.

56 Vgl. Hans Herbert von Arnim, a.a.O. (Fn. 53).

57 So auch Martin Morlok, in: Horst Dreier (Hrsg.), Grundgesetz-Kommentar, München 2010, Art. 38 Rdnr. 52; Hans Meyer, in: Josef Isensee / Paul Kirchhof (Hrsg.), Handbuch des Staatsrechts, Heidelberg 2005, Bd. III, $\$ 45$ Rdnr. 17.

58 So auch Il Sun Hong, a.a.O. (Fn. 6), S. 35. 
die Schlussfolgerung nahe, dass der Anwendungsbereich der $\$ \$ 107 \mathrm{ff}$. StGB im Einklang mit $₫ 108$ d StGB auf parteiinterne Wahlen ausgedehnt ist, denn die Kandidatenaufstellung ist auch staatsrechtlich Teil der Wahl. Der Wortlaut des $\$ 108 \mathrm{~d}$ StGB schließt nicht aus, dass mit dem Begriff der Wahl nicht nur die staatliche Vorbereitung, die Abstimmung und die Feststellung des Wahlergebnisses, sondern auch die Aufstellungsverfahren erfasst werden.

Das strafrechtliche Analogieverbot steht dem nicht entgegen. Nach Art. 103 Abs. 2 GG - Gewährleistung hinreichend bestimmter Straftatbestände, Vorhersehbarkeit strafbedrohten Verhaltens für jedermann - ist sowohl die rückwirkende Anwendung neu geschaffener Straftatbestände als auch die Strafbegründung im Wege der Analogie oder des Gewohnheitsrechts verboten..$^{59}$ Neben dem Anknüpfen der $\$ \$ 107 \mathrm{ff}$. StGB an bereits zeitlich vor dem Wahlgang liegende Handlungen dürfte aber vielmehr entscheidend sein, dass der Gesetzgeber durch die Strafbewehrung der Abgabe einer eidesstattlichen Versicherung deutlich gemacht hat, dass er an den Ablauf einer Aufstellungsversammlung klare, auf den verfassungsrechtlichen Wahlgrundsätzen beruhende Anforderungen stellt, die denen des Wahlgangs am Wahltag nicht nachstehen. Sinn und Zweck dieser Regelung ist es nicht nur, ein Protokoll mit verstärkter Beweiskraft zu erlangen, sondern auch die Einhaltung der Wahlrechtsgrundsätze durch den Versammlungsleiter sowie zwei weitere verantwortlich benannte Personen sicherzustellen. Ersichtlich wird daraus, dass die Einhaltung der verfassungsrechtlichen Wahlrechtsgrundsätze bei Aufstellungsversammlungen unter einem besonderen und deutlich stärkeren Schutz als bei sonstigen parteiinternen Wahlen steht. Die parteiinterne Aufstellungsversammlung wird durch den Gesetzgeber als strafrechtlich schützenswert angesehen und bereits strafrechtlich geschützt. Insbesondere durch die Beteiligungsregelung des $\$ 160$ StGB wird klargestellt, dass nicht nur das Verhalten der Versicherer an Eides statt strafrechtlich sanktioniert werden kann, sondern auch Dritte keinen Einfluss auf den Ablauf einer Aufstellungsversammlung nehmen dürfen.

Auch der Schutzzweck der $\$ \$ 107 \mathrm{ff}$. StGB legt es nahe, die Kandidatenaufstellung in die Tatbestände einzubeziehen. Sinn und Zweck der Wahlstraftaten liegt vornehmlich in der Wahrung der Authentiziät von Wahlen und Abstimmungen in Form ihres unbeeinträchtigten Ablaufs. ${ }^{60}$ Damit soll die in staatlichen Angelegenheiten gewährleistete Willensbildung und verfassungsrechtlich gesicherte Demokratie in ihren Erscheinungsformen geschützt werden. ${ }^{61}$ Die freie und geheime Wahl ist unabdingbar für die Existenz des Staates als Demokratie. Damit wird deutlich, wie besonders wichtig - insbesondere aufgrund der geschichtlichen Erfahrungen - der strafrechtliche Schutz dieses Rechtsgutes ist. Überdies besteht der Schutzzweck der $\$ \$ 107 \mathrm{ff}$. StGB nicht nur in einem Allgemeinrechtsgut, sondern hat auch einen Individualcharakter, da das Wahlrecht ein Menschenrecht ist und der Wählende ein Interesse daran hat, dass seine Stimme auch einen gleichen Wert hat. ${ }^{62}$

59 Vgl. Stefanie Schmahl, in: Bruno Schmidt-Bleibtreu / Hans Hofmann / Axel Hopfauf(Hrsg.), Kommentar zum Grundgesetz, Köln 2011, Art. 103 Rdnr. 24, 36.

60 Vgl. Georg Bauer / Duscha Gmel, a.a.O. (Fn. 28), Vor \$\$ 107 Rdnr. 1; Dominik Härtel, a.a.O. (Fn. 2), S. 18. Differenziert bei Wolfgang Wohlers, in: Urs Kindhäuser / Ulfried Neumann / HansUlrich Paeffgen (Hrsg.), Nomos-Kommentar zum Strafgesetzbuch, Baden-Baden 2010, Vor \$\$ 105 Rdnr. 1.

61 Vgl. Reinhart Maurach / Friedrich-Christian Schröder / Manfred Maiwald, Strafrecht Besonderer Teil, Bd. 2, Heidelberg 2005, $\$ 86$ Rdnr. 1.

62 Vgl. ebenda; BVerfGE 121, S. 266 ff., Rz. 91 ff.; Gerhard Wolf, a.a.O. (Fn. 2), S. 166 f. Das BVerfG spricht von einem „Bürgerrecht auf Teilnahme an der Wahl“, BVerfGE 89, S. 243 ff., S. 251. 
Bei der Aufstellung von Kandidaten handelt es sich um notwendige und mit der Wahl selber untrennbar verbundene Vorbereitungshandlungen, die unmittelbar das passive Wahlrecht berühren. Um richtige Wahlergebnisse zu erzielen, ist es auch erforderlich, dass der richtige Kandidat zur Wahl steht, denn mit der Aufstellung des falschen Kandidaten wird bereits die Ursache für ein falsches Wahlergebnis gelegt. „Wird bei den Aufstellungsverfahren der Kernbestand an Verfahrensgrundsätzen, der sich aus dem Verfassungsrecht und den Wahlgesetzen ergibt, nicht eingehalten, so wird die demokratische Legitimationswirkung staatlicher Wahlen schlechthin in Frage gestellt und begründet die Gefahr der Verfälschung des demokratischen Charakters der Wahl bereits in ihrer Grundlage."63 Dabei kann es nicht darauf ankommen, ob das falsche Wahlergebnis beispielsweise durch eine Veränderung der abgegebenen Stimmen am Wahltag entstanden ist oder ob seine Ursache bereits im Rahmen der Kandidatenaufstellung gelegt wurde, da selbst die Abgabe der Stimmen durch die Wähler nicht mehr zu einem richtigen Wahlergebnis führen kann. Mit der Norm des $\$ 107 \mathrm{~b}$ Abs. 1 Nr. 4 StGB hat der Gesetzgeber deutlich gemacht, dass er auch darauf Wert legt, dass wählbare und damit richtige Kandidaten zur Wahl stehen, denn auch die Wahl eines falschen Kandidaten kann die Wahl verfälschen; schließlich kommt es auf jede einzelne Stimme an, wie sich aus den anderen Tatbeständen des $₫ 107 \mathrm{~b}$ Abs. 1 Nr. 1-3 StGB ergibt.

Auch wenn es sich bei der Kandidatenaufstellung auf den ersten Blick um einen parteiinternen Akt handelt, da ja die Parteien "Veranstalter" sind, ist doch der verfassungsrechtliche Auftrag aus Art. 21 Abs. 1 GG, aus dem sich das Recht der Parteien zur Beteiligung an Wahlen ableitet, nicht außer Acht zu lassen. Ohne die Aufstellung von Kandidaten wäre die Mitwirkung an der politischen Willensbildung durch die Parteien maßgeblich eingeschränkt. Und gerade die Wahl von Parlamentsmitgliedern ist in einer repräsentativen Demokratie von zentraler Bedeutung für die Berücksichtigung der politischen Interessen und die Vertretung des Volkes. Umso mehr kann die Notwendigkeit bejaht werden, diesen „vorgeschalteten" Bereich auch strafrechtlich über die Versicherung an Eides statt nach $\$ 21$ Abs. 6 BWahlG in Verbindung mit $\$ 156$ StGB hinaus zu schützen.

\section{Vollendungszeitpunkt}

Für den Vollendungszeitpunkt bei einer Anwendung der $\$ \$ 107 \mathrm{ff}$. StGB auf Aufstellungsversammlungen kommen grundsätzlich vier unterschiedliche Zeitpunkte in Betracht: das Ende der Aufstellungsversammlung, das Unterschreiben der Dokumente für die Einreichung der Wahlvorschläge, die Einreichung der Wahlvorschläge bei den zuständigen Wahlleitern sowie die Zulassungs-/Ablehnungsentscheidung durch die Wahlleiter/-ausschüsse.

Hilfreich zur Klärung dieser Frage dürfte ein Blick auf den Ablauf des sich an die Aufstellungsversammlung anschließenden Verfahrens sein: Der Erfolg bei einer Manipulation im Aufstellungsverfahren tritt ein, wenn das Ergebnis der Auszählung der Aufstellung bekannt gegeben wird. Die folgenden Schritte laufen schon fast automatisch ab, sie sind nur

63 BVerfGE 89, S. 243 ff., S. 252; SächsVerfGH, a.a.O. (Fn. 36), S. 270. So auch bereits Klaus Otto Nass, Die verfassungsrechtlichen Wahlrechtsgrundsätze bei der der Aufstellung von Parteikandidaten für Bundestagswahlen, in: Hans Schneider (Hrsg.), Im Dienst an Recht und Staat, Festschrift für Werner Weber, Berlin 1974, S. 311 - 324, S. 316; Rüdiger Wolfrum, Die innerparteiliche Ordnung nach dem Parteiengesetz, Berlin 1974, S. 180. 
eine Konsequenz der Manipulation, auf die der Täter nicht zwingend Einfluss hat. Naturgemäß kommt es ihm darauf an, dass der erfolgreich manipulierte Wahlvorschlag eingereicht wird; in aller Regel geschieht dies dann jedoch ohne sein Zutun, eine weitere Handlung seinerseits ist nicht mehr erforderlich. Ein Rücktritt des Täters ist nach Abschluss der Versammlung bis zur Entscheidung über die Zulassung des Wahlvorschlags nur möglich durch aktives Tun. ${ }^{64}$ Dies spricht dafür, den Vollendungszeitpunkt mit dem Eintritt des Erfolgs der unmittelbaren Handlung, der Manipulation, zusammenzulegen und eine Vollendung mit dem Ende der Aufstellungsversammlung anzunehmen. ${ }^{65}$

Anwendbare Tatbestände auf die Fallkonstellationen

Die verschleppte Nicht-Aufnahme von Neumitgliedern erfüllt nicht den Straftatbestand des $\$ 107$ b Abs. 1 Nr. 3 StGB. Nach $\$ 21$ Abs. 1 Satz BWahlG sind alle Personen, die zum Zeitpunkt der Aufstellungsversammlung Parteimitglied sind, wahlberechtigt. Wer noch nicht aufgenommen ist, kann also nicht mit aufstellen; die Gründe hierfür können vielfältig sein. Dies ist allerdings eine Angelegenheit die den Parteien überlassen ist und keine Frage des Wahlrechts. Auseinandersetzungen über Mitgliedsrechte sind in erster Linie intern zu klären; gegebenenfalls hat die Partei Aufnahmefristen satzungsrechtlich festgelegt. ${ }^{66}$

Die Fälschung von Aufnahmeanträgen beziehungsweise das Erschleichen der Wahlunterlagen könnte eine Strafbarkeit nach $\$ 107$ a sowie $107 \mathrm{~b}$ Abs. 1 Nr. 1 StGB begründen. Außerdem könnte die Manipulation bei der Wahl selber durch Hinzufügen beziehungsweise Wegnehmen oder Ändern der Stimmzettel den Tatbestand des $\$ 107$ a StGB erfüllen. Ob und wie diese Tatbestände verwirklicht sind oder ob beispielsweise die $\$ \$ 108,108$ b StGB greifen, hängt stark vom Vorgehen des Täters im Einzelfall ab. Hinsichtlich der Beteiligungsformen durch mögliche Mittäter, Anstifter und Gehilfen gelten die allgemeinen Regeln. Aufgrund des unterschiedlichen Schutzzwecks stehen die $\$ \$ 107$ ff. StGB in Idealkonkurrenz zu den $\$ \$ 267$ ff. StGB. ${ }^{67}$

\section{Strafrechtlicher Schutz der Kandidatenaufstellung: Mahnung an die Parteien}

Parteiinterne Wahlen sind kein strafrechtsrechtsfreier Raum. Allerdings ist nicht jede Wahl strafrechtlich gleich zu werten, der Schutz der $\$ \$ 107$ ff. StGB gilt lediglich für die Aufstellung von Bewerbern zu öffentlichen Wahlen. Der Gesetzgeber hat diese Versammlungen bereits durch die Versicherung an Eides statt gemäß $\$ 21$ Abs. 6 BWahlG in Verbindung mit $\$ 165$ StGB unter strafrechtlichen Schutz gestellt.

64 Die in $\$ 21$ Abs. 4 BWahlG einem Vorstand eingeräumte Möglichkeit, eine Aufstellungsversammlung wiederholen zu lassen, kann nicht zu einer anderen Bewertung führen, da diese Möglichkeit satzungsabhängig ist. Siehe hierzu LG Köln, NVwZ 2005, S. 358.

65 Hinsichtlich des Beginns der Strafbarkeit bestehen keine Besonderheiten, es gelten die allgemeinen Regeln zur Versuchsstrafbarkeit in Verbindung mit den jeweiligen Strafrechtsnormen.

66 Vgl. Jörn Ipsen, a.a.O. (Fn. 21), \$10 Rdnr. 4.

67 Vgl. zusammenfassend Dominik Härtel, a.a.O. (Fn. 2), S. 159. 
Dies ist angesichts der Bedeutung der Aufstellungsversammlungen für Wahlen nicht nur konsequent, sondern auch sinnvoll. ${ }^{68}$ Die früher diskutierte Gefahr, dass hierdurch eine Gefährdung des demokratischen Staatsaufbaus eintreten würde, indem die Strafverfolgungsbehörden - eventuell sogar auf Weisung des Justizministers - in Interna der (Oppositions-)Parteien eindringen ${ }^{69}$, dürfte angesichts der sich verändernden Medienlandschaft als überholt gelten. Die Veröffentlichungen von WikiLeaks, beispielsweise das fast zwanzigseitige „Dossier“ bei Wikipedia über die Münchener CSU-Affäre und der mit dem „Medienprojektpreis 2010 für kritischen Journalismus“ ausgezeichnete Blog „Wir-in-NRW-Blog. de“ zeigen, dass es ein deutliches Interesse der Bevölkerung an solchen Informationen gibt und Journalisten auch bereit sind, diese zu recherchieren und zu veröffentlichen, ohne auf eine Verlagszeitung angewiesen zu sein. Diese Entwicklung dürfte nicht mehr aufzuhalten sein und Entscheidungen auf allen Ebenen zukünftig verstärkt mit beeinflussen.

Die Erfahrungen mit Wahlprüfungen durch Parlamente und Gerichte bei der Vielzahl von Wahlen seit Gründung der Bundesrepublik zeigen zudem, dass mit der bestehenden Kontrolle verantwortungsvoll und -bewusst umgegangen wird. Der bekannteste „Skandal“ dürfte die Entscheidung des Hamburger Verfassungsgerichts von 1993 sein, größere Skandale sind weitestgehend ausgeblieben. Eine Kooperation mit der Staatsanwaltschaft ist daher gut vorstellbar, denn die Sitzungen der Wahlausschüsse sind ohnehin öffentlich. Hier dürfte jedoch eine intensivere Zusammenarbeit zwischen den jeweiligen Wahlleitern und Staatsanwälten wünschenswert sein, da der Eindruck besteht, dass die Staatsanwaltschaften erst aufgrund einer öffentlichen Berichterstattung tätig werden.

Das sich wandelnde Wahlverhalten trägt dazu bei, dass immer mehr Parteien entstehen, deren Organisationsgrad mitunter fragwürdig erscheint. ${ }^{70}$ Durch das Bewusstsein strafrechtlichen Schutzes der Aufstellungsversammlung dürfte den Parteien - gleich welcher Ausrichtung sie sind - immer wieder deutlich werden, dass sie einen verfassungsrechtlichen Auftrag haben.

Die Urkundendelikte nach $\$ \$ 267 \mathrm{ff}$. StGB sind demgegenüber auf alle parteiinternen Wahlen und Abstimmungen anwendbar. Die Tatbestandsverwirklichung hängt jedoch vom Einzelfall ab; die in einer Wahlurne enthaltenen Stimmzettel sind allerdings mangels Erkennbarkeit der Aussteller keine zusammengesetzte Urkunde. Da es sich bei Vorstandswahlen und Abstimmungen um rein parteiinterne Vorgänge handelt, erscheint dieser strafrechtliche Schutz ausreichend.

68 Vgl. andere Auffassung ebenda, S. 196; Gerhard Wolf, a.a.O. (Fn. 2), S. 102.

69 Vgl. Josef Schafheutle (Vertreter des Justizministerium) und Ludwig Schneider (FDP) bei den Verhandlungen der Kommission zur Erstellung eines neuen Strafgesetzbuches von 1954 bis 1959, in: Niederschriften über die Sitzungen der Großen Strafrechtskommission, a.a.O. (Fn. 2), S. 289 f., S. 292; anderer Auffassung Paul Bockelmann (Staatsrechtler), in: ebenda, S. 292 mit Hinweis auf die Position des Bundesinnenministeriums.

70 An der Bundestagswahl 2009 nahmen 28 Parteien teil, ebenso viele Vereinigungen wurden vom Bundeswahlausschuss nicht als Parteien anerkannt. Vgl. Brigitte Gisart, Endgültiges Ergebnis der Wahl zum 17. Deutschen Bundestag am 27. September 2009, in: Wirtschaft und Statistik 2009, S. $1063-1079$, S. 1063. 